\title{
A Scaled Electronically-configurable Multichannel Recording Array
}

\author{
J JI, $K$ NAJAFI and $K$ D WISE
}

Center for Integrated Sensors and Crcuts, Department of Electrical Engineering and Computer Sctence, Unversity of Michigan, Ann Arbor, MI 48109-2122 (US A)

\begin{abstract}
This paper describes a multıchannel microelectrode array capable of recording single-unit neural activity in the central nervous system The probe features 32 recording sites on a scaled shank less than $50 \mu \mathrm{m}$ wide On-chıp CMOS crcuitry implements signal amplification, multiplexing, and selftesting on eight active channels selected from among the 32 sites The circuitry has a power dissipation of $3 \mathrm{~mW}$, an active area of $25 \mathrm{~mm}^{2}$, and requires only three external leads It utılizes bidirectional signal transmission over the output data lead for signal output and for channel selection
\end{abstract}

\section{Introduction}

Silicon micromachıned multıelectrode recording probes are important for studying information processing in neural systems and for eventual use in a variety of neural prostheses $[1,2]$ The probes offer very small precisely-controlled dimensions, the ability to utilize a large number of electrodes with minimal tissue damage, compatibility with on-chip signal processing, and biocompatibility Using such probes, new information has been obtained with regard to the operation and functional organization of neural structures [2], however, the distance over which cell activity can be recorded is typically limited to about $100 \mu \mathrm{m}$ in the cortex, so that proper positioning of the probe with respect to the active cells is essential In order to adequately sample a volume of tissue, two- and three-dimensional recording will eventually be required with many more recording sites than have been possible in the past This paper reports the process technology and functional design for a second-generation recording probe which offers scaled geometries, a high-performance CMOS process, all static circuitry, and the ability to position electronically the active recording sites in tissue in order to optimize electrode-cell coupling The probe can be organized as either a single 32-electrode shank or as several laterally- spaced multselectrode shanks Thus, two-dmensional recording is possible

\section{Probe Structure and Process Technology}

The overall probe structure, as shown in Fig 1, consists of multiple recording sites supported by a micromachined silicon substrate which has been scaled down in size to minimize tissue damage The probe is fabricated with a technology that combines deep-boron diffusion/boron etch-stop techniques with a high-performance $3 \mu \mathrm{m}$, p-well, double-poly CMOS process The intended probe shape is first defined using a deep boron diffusion, and the circuitry is then fabricated in CMOS with the metalization step being shared for both the circuitry and the electrode conductors After passivation with LPCVD silicon dioxide and nitride layers, the recording site and bonding pad vas are opened and inlayed with gold Finally, the wafer substrate is dissolved in EDP, leaving only the probes Except for the final etch, all the process steps are standard silicon IC processes With this technology, probes can be made in batch with very high yields and can be designed with arbitrary two-dimensional shapes $(\mathrm{e} g$, multiple

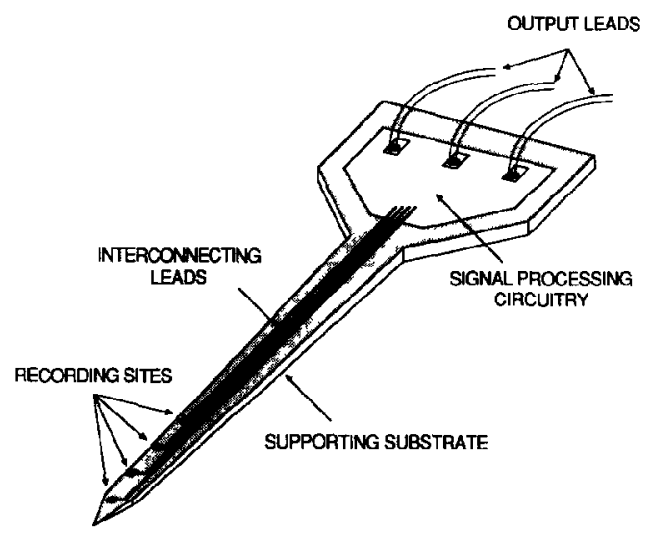

Fig 1 A multielectrode probe for chronic intracortical neural recording

(C) Elsevier Sequoia/Printed in The Netherlands 


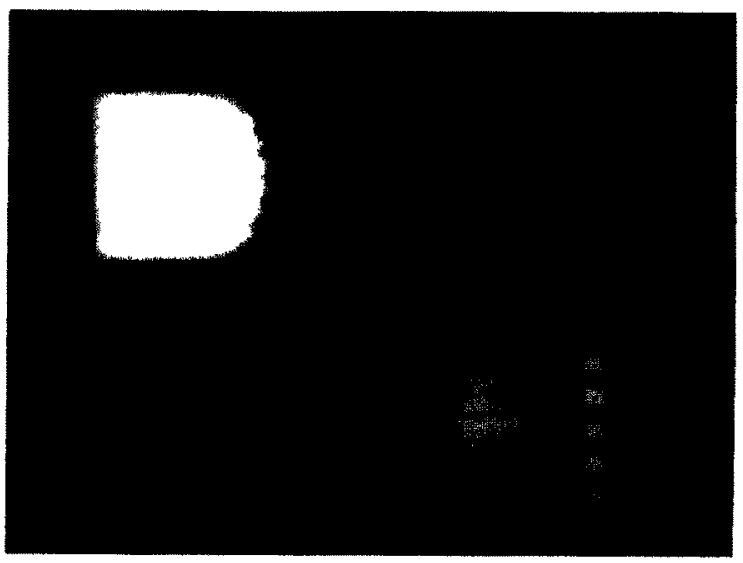

Fig 2 A six-shank scaled probe structure capable of lateral as well as vertıcal recording

shanks) and very small precisely-controlled dimensions The single-shank design supports 32 recording electrodes, with feature sizes of $1 \mu \mathrm{m}$ for the interconnects on the shank and appropriately scaled shank dimensions [3] Figure 2 shows a six-shank scaled probe for lateral as well as vertical (in depth) recording Each shank is $30 \mu \mathrm{m}$ wide, with a center-to-center shank spacing of $100 \mu \mathrm{m}$ These probes enter the tissue easily with minimal dimpling of the cortical surface and sufficient strength to penetrate the p1a arachnoid membrane

\section{Functional Design for On-chip Signal Processing}

Figure 3 shows a block diagram of the secondgeneration probe electronics, contrasting it with the first-generation design The new probe contains preamplifiers, an analog multiplexer, and self-test circuitry as in the previous design [1], but these circuit blocks have been redesigned in CMOS A 32-to-8 front-end selector and an input/ output (I/O) controller have also been added Although 32 electrodes are provided to cover a large recording field, only eight signal channels are used at any one time By selecting only those sites which best record the activity present, the external processing of inactive channels is avoided and chip area and power dissipation are minimized The CMOS preamplifiers provide a gain of 300 and a bandwith from $100 \mathrm{~Hz}$ to $10 \mathrm{kHz}$ The I/O controller allows the external data lead to be used in either of two modes in the input mode, a digital data stream is fed into the probe to program the front-end selector, in the output mode, the time-multiplexed analog neural signals are transmitted to external circuitry Figure 4 shows the different signals transmitted over the I/O data line Clock, data and control signals are superimposed on the data line during the loading of the 16-bit selector setting, allowing this sensor to be externally programmed without increasing the external lead count On-chip self-test circuitry is activated by a pulse on the VDD line Thus, the
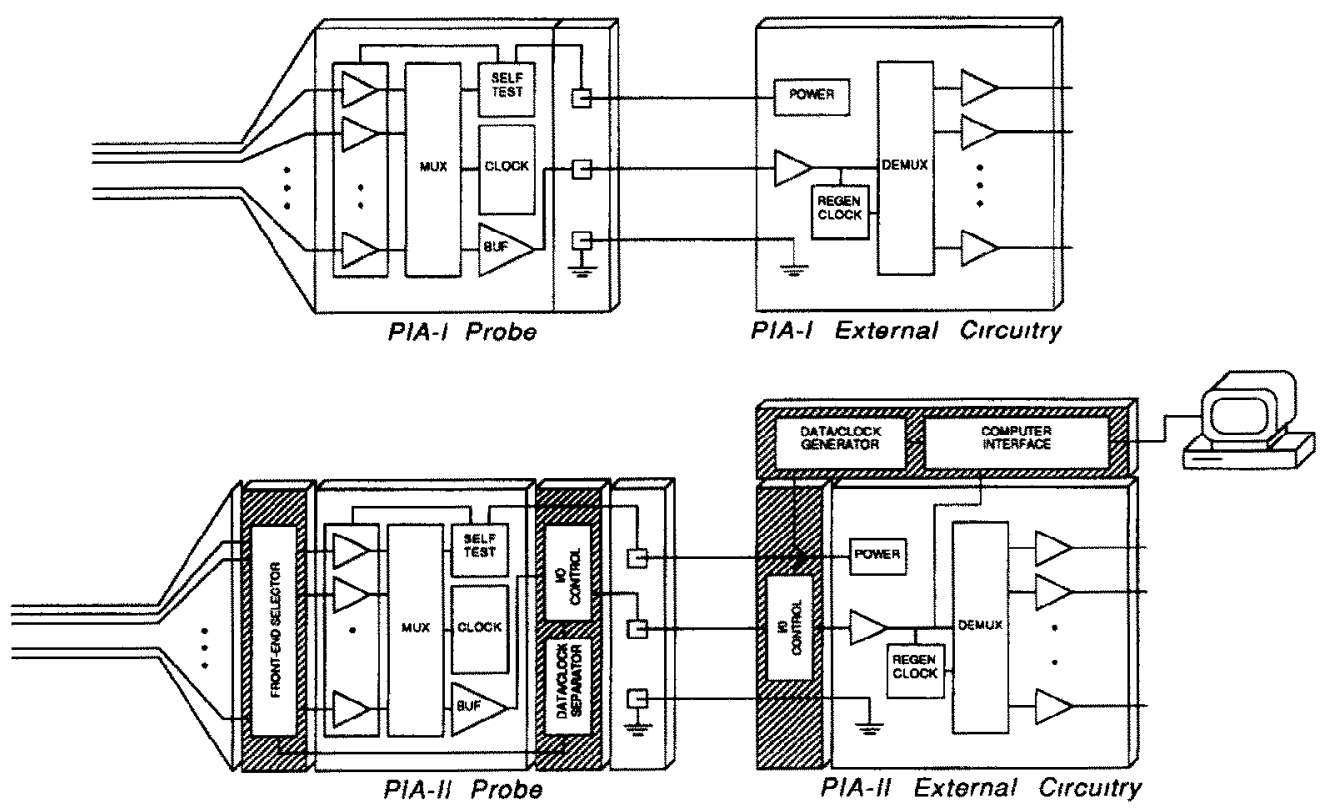

Fig 3 Comparison of the circuitry for the first- and second-generation probe designs 


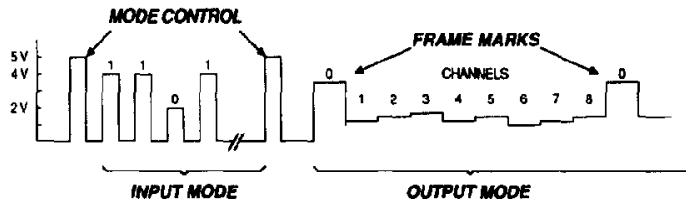

Fig 4 Detalled diagram of signaling on the $1 / O$ line

sensor implements six functions on three external leads (one of which is ground) power, reset, self-test, clock, digital input data and analog output Circuit functionality has been verified through detailed simulations and through actual fabrication using the chip foundry MOSIS Fabrication on the first full second-generation active probes is now under way

\section{Conclusions}

Figure 5 offers a comparison of the first- and second-generation probe designs This secondgeneration probe offers a significant reduction in substrate size, a substantial increase in the number of possible recording sites, and no change in the number of external leads It represents a significant step forward in the development of electrode arrays which can chronically interface with neural structures in two- and three-dimensions for both research and prosthetic applications

\section{Acknowledgements}

The authors wish to express their appreciation to Dr F T Hambrecht and Dr W Heetderks of the National Institutes of Health for their support and encouragement in this work This work was supported by the National Institutes of Health under contract NIH-NINCDS-N01-NS-72397

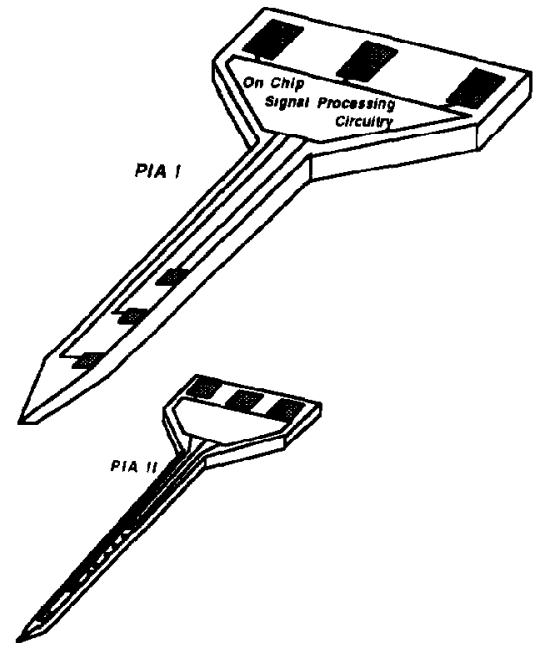

Fig 5 Characteristics of the first- and second-generation active probe designs

\begin{tabular}{lll}
\hline Parameter & PIA-I & PIA-II \\
\hline No of recording sites & 10 & 32 \\
Feature size & $6 \mu \mathrm{m}$ & $3 \mu \mathrm{m} / \mathrm{I} \mu \mathrm{m}$ \\
Technology & E/D NMOS & CMOS \\
Thickness & $15 \mu \mathrm{m}$ & $15 \mu \mathrm{m}$ \\
Shank length & $32 \mathrm{~mm}$ & $32 \mathrm{~mm}$ \\
Mid-shank width & $120 \mu \mathrm{m}$ & $50 \mu \mathrm{m}$ \\
Total displaced volume & $61 \mathrm{E}-6 \mathrm{~cm}^{3}$ & $24 \mathrm{E}-6 \mathrm{~cm}^{3}$ \\
No of leads & 3 & 3 \\
Circuit area & $19 \mathrm{~mm}^{2}$ & $25 \mathrm{~mm}^{2}$ \\
Power dissipation & $5 \mathrm{~mW}$ & $3 \mathrm{~mW}$ \\
Transistor count & 480 & 890 \\
\hline
\end{tabular}

\section{References}

$1 \mathrm{~K}$ Najafi and K D Wise, An implantable multielectrode array with on-chip signal processing, IEEE $J$ Solid-State Circuits, SC-2I (1986) 1035-1044

2 K L Drake, K D Wise, J Farraye, D J Anderson and S L BeMent, Performance of planar multisite microprobes in recording extracellular single-unit intracortical activity, IEEE Trans Biomed Eng , BME-35 (1988) 719-723

$3 \mathrm{~K}$ Najafi, J Ji and $\mathrm{K}$ D Wise, Multichannel intracortical recording microprobes scaling limrtations, device characteristics, and circuit encapsulation, Proc 4th Int Conf SoltdState Sensors and Actuators (Transducers '87), Tokyo, Japan, June 2-5, 1987, pp 65-68 